FedUni ResearchOnline

http://researchonline.federation.edu.au

This is a Submitted Manuscript of an article published by Taylor \& Francis in Physical Education and Sport Pedagogy on 25/04/2016, available online: 


\section{Caution Regarding Exergames: A Skill Acquisition Perspective}

Scott J. Pedersen, Paul D. Cooley, \& Vaughan J. Cruickshank

Faculty of Education, University of Tasmania, Launceston, Australia

Corresponding Author:

Scott J. Pedersen, Ph.D.

University of Tasmania

Locked Bag 1307

Launceston TAS 7250

Australia

scott.pedersen@utas.edu.au

Office: +6163243554

Fax: +61 63243679

Running Header: EXERGAMES AND SKILL ACQUISITION

Word Count: 6522 


\section{Caution Regarding Exergames: A Skill Acquisition Perspective}

Background: The advent of technology use in physical education is upon us. But the implications of using exergames as a substitute for traditional physical education instruction for some students raise questions. Although exergames have the potential to increase energy expenditure and motivation in some children, it is less clear whether they can provide skill acquisition benefits that are similar to those found in traditional physical education.

Purpose: In a previous experiment from our laboratory we found that deliberate practice can significantly reduce the planning time required for lateral arm movements. The purpose of this study was to determine if exergames can produce a similar effect, by reducing the processing time required for children to initiate arm movements to the contralateral and ipsilateral space.

Participants and setting: Thirty children (boys=15, girls=15), between the ages of 7 and 12 years, participated in a pre and posttest each taking 30 minutes and one 30 minute treatment session in a university laboratory.

Research design: A repeated measures design was employed to test the effects of deliberate laterality practice on processing speed. Children were randomly assigned $(n=10)$ to either a Nintendo Wii tennis contralateral movement experimental group, Nintendo Wii bowling ipsilateral movement experimental group, or hand-held video game control group. Each child participated in one 30 minute treatment session.

Data collection: Upper extremity choice reaction time was measured through 27 goal-directed aiming movements for each arm separately, during the pretest and posttest. The stimulusresponse trials occurred in three randomly presented directions (ipsilateral, contralateral, and midline). 
Data analysis: A 3 (treatment group) $\times 2$ (age group) $\times 2$ (test $) \times 3$ (direction) mixed design analysis of variance with repeated measures on the last two factors was used to test for significant differences, with an alpha level set at 0.05 .

Findings: There were no significant treatment effects on reaction time across all groups indicating that a short bout of exergame training was unsuccessful in improving lateral movement processing.

Conclusions: Deliberate laterality practice using exergames did not improve the motor processing speed of lateral arm movements in the same manner of traditional physical activity as indicated by our previous research. Explanations as to why exergames do not exhibit the same positive transfer for skill acquisition as traditional physical education instruction are discussed within this paper.

Keywords: exergames; skill acquisition; deliberate practice; laterality; children 


\section{Introduction}

Sedentariness is connected to a range of adverse health conditions within the population. Causes of sedentariness are varied, but within young people, the growing prevalence of video gaming is often considered a contributing factor despite evidence to the contrary (Lager and Bremberg 2005; Marshall et al. 2004; Sun 2012; Sheehan and Katz 2013). As a by-product of better technology, video game manufacturers have been able to increase the physical and cognitive fidelity of their sport simulation tasks (Chandler, Anthony, and Klinger 2009). Games now give users a similar experience to the real sport skill. For example, with the Nintendo Wii players are required to move the game controller, a handheld device, through space as if they were actually implementing a piece of sporting equipment such as a tennis racket or a bowling ball. Moreover, players can apply strategy to achieve better results such as changing the approach position. Games that have a degree of similar physical and cognitive fidelity are defined as exergames (Hansen and Sanders 2008). Physical educators (e.g., Staiano and Calvert 2011; Yang, Smith, and Graham 2008) recognising the motivational impact of exergames have co-opted their use within physical education lessons as a means of shifting students from sedentariness to low level physical activity (Hayes and Silberman 2007), improving health (e.g., Bailey and McInnis 2011; Graf et al. 2009) and as a means of re-engaging students (Widman, McDonald, and Abresch 2006). Playing sport is a central feature of the Australian national curriculum (ACARA 2015), where a portion of the curriculum focuses explicitly on students developing the psychomotor movement skills required to competently and confidently participate in sport based activity. Despite the increased use of exergames in physical education there is some data (O'Leary et al. 2011) that there is little transfer of fidelity from gaming to applying their skills to a sport specific environment. To add to this literature we designed a follow-up study to our previous 
work (Pedersen 2014) to determine if exergames are as effective for promoting the development of processing lateral arm movements as traditional physical activity.

For exergames to be deemed as a useful mode for psychomotor skill development there should be a positive transfer effect from gaming to training. Researchers that have plotted exergame and traditional game experimental groups against one another have been unable to demonstrate that exergames are as effective for psychomotor skill development as the more traditional activities (Brumels et al. 2008; Vernadakis et al. 2012). Several studies in this area (e.g., Kliem and Wiemeyer 2010; Sheehan and Katz 2013) have focused on developing balance ability primarily due to the availability of the Nintendo Wii balance board apparatus for use with several of the Nintendo Wii Fit exergames. While these results were interesting, the balance tests used were not a stringent indicator of psychomotor skill. Pedersen et al. (2005) pointed out that psychomotor measurements that do not include temporal constraints allow an assessment savvy participant to skew the desired outcome of the measurement. Thus, utilising a choice reaction time (RT) test to measure information processing ability might be a more suitable measurement to detect differences in psychomotor skill development. Moreover, RT has been identified as an important variable of balance (Bisson et al. 2007; Woollacott and Sumway-Cook 2002). Thus, in the current study we elected to use a more temporally sensitive measure of this processing speed ability through a measure of upper extremity choice RT.

Information processing, as measured by RT, can be improved in children with deliberate practice strategies (Hale, Fry, and Jessie 1993; Kail 1991). In a previous study we (Pedersen 2014) extended this research by investigating whether a short bout (30 minutes) of deliberate laterality practice effected the sensory-motor processing of lateral movements in children. More specifically we investigated whether or not a short bout of deliberate practice using a tailor-made progression from simple to complex ball bouncing movements, adapted 
from the commercially available Bal-A-Vis-X training programme (Hubert 2001), could improve the lateral processing times associated with these movements. These ball bouncing treatments were designed using specificity of training principles (Reilly et al. 2009), and tenants of Ericsson, Krampe, and Tesch-Romer's (1993) theory of deliberate practice on expert performance. We found that children who performed activities that required arm movements across their body's midline significantly improved their ability to initiate more complex contralateral upper extremity responses. Similarly, the ipsilateral ball bouncing group exhibited significantly shorter RTs in the simpler ipsilateral direction after the treatment. These findings supported the use of deliberate practice of traditional physical activity to invoke positive transfer towards the psychomotor development of processing lateral movements in children. In the current study, we are investigating if these findings can be extended to a short bout of deliberate practice using exergame activities. Thus, we replicated the Pedersen (2014) study by only changing the treatment groups from traditional ball bouncing games to exergames. The purpose of this study was to investigate the deliberate practice effect of exergames on the initiation speed of lateral arm movements in children. Based on our previous findings we hypothesised that exergame training based on deliberate lateral arm movements to the contralateral and ipsilateral space will significantly reduce contralateral and ipsilateral choice reaction time scores, respectively. . It is recommended that the reader review the significant results of this previous study in order to better understand the findings discussed within this comparative investigation.

\section{Methods}

\section{Participants}

Thirty boys and girls $(M$ age $=9.73 \pm 1.79$ years, range $=7$ years 1 month to 12 years 5 months) were randomly selected from several communities (rural and urban) in Tasmania, Australia to participate in this study. Participants and their parent/guardian provided informed 
consent in accordance with guidelines established by the university's institutional review board for human research. Potential participants were made aware of this study by local newspaper, webpage, and flyer advertisements soliciting children in the identified age range. Children were pre-screened by phone interview with their parent/guardian to ensure no participants received any special services for developmental, physical, sensory, or cognitive disabilities. All participants were right-handed according to Bryden's (1977) shortened fiveitem version of the Oldfield handedness questionnaire.

After screening, all children were randomly assigned without replacement to one of three treatment groups. The first two groups were experimental. The Wii tennis contralateral (WTC) movement experimental group ( mean age=116.60+/-22 months; boys=4, girls=6) performed a tennis simulation exergame that required contralateral arm and leg movements for each groundstroke performed. The Wii bowling ipsilateral (WBI) movement experimental group ( mean age=119.70+/-26 months; boys=6, girls=4) performed only ipsilateral arm and leg extremity movements during a bowling simulation exergame. Finally, the control group ( mean age $=113.80+/-19$ months; boys=5, girls=5) played a hand-held video game in which the children did not perform any lateral arm or leg movements. These groups were not significantly different from each other based on age or sex. To test for developmental age differences participants were also categorised into younger (7-9 year olds; mean age $=97.87+/-8.99$ months; $\mathrm{n}=15)$ and older $(10-12$ year olds; mean age $=135.53+/-13.27$ months; $\mathrm{n}=15$ ) cohorts.

Before and after the 30-minute treatment (experimental and control) session all children were assessed on a custom made upper extremity, three-choice response time apparatus that required the participant to perform goal-directed arm movements to either a contralateral stimulus across the midline, a midline stimulus, or an ipsilateral stimulus that 
did not require a midline crossing arm movement. Each participant was tested and trained on an individual basis, on the same day in an isolated room with adequate lighting.

\section{Apparatus}

A custom-built aiming apparatus was used to obtain upper extremity choice response time data (figure 1). This apparatus was a table mounted wooden board $(60 \times 70 \mathrm{~cm}$ and 1 cm thick) consisting of a releasable microswitch (i.e., start pad) and three target pads with corresponding red light-emitting diodes (LED) positioned directly behind each target pad. The start pad was situated in the bottom centre of the board, with one target pad directly in front, one to the right, and one to the left. The start pad and three target pads were $2.5 \mathrm{~cm}$ wide and constructed out of durable plastic mounted in a 3 X $2 \mathrm{~cm}$ metal, circular frame. The red LEDs were mounted in $3 \times 2 \mathrm{~cm}$ metal frames and placed $7 \mathrm{~cm}$ behind the corresponding target pad. All three target pads were at an equal distance $(33.5 \mathrm{~cm})$ from the start pad, with the right and left targets positioned $45^{\circ}$ from midline to allow the participant to have an equal view of all three red LEDs while fixated at the centre of the board. [Figure 1 near here]

An auditory warning signal was housed in a $13 \times 9 \mathrm{~cm}$ box resting on the top centre of the board to notify the participant to initiate each trial. On the proximal surface of this box was a yellow LED, located $20 \mathrm{~cm}$ directly anterior to the midline target LED, which served as a catch trial stimulus. A portable laptop computer interfaced through a standard parallel printer port contained custom-built computer software that generated randomised blocks of trials and stimulus presentations, and collected separate values for reaction time (RT: time from presentation of stimulus light to release of pressure from the start pad) and movement time (MT: time from release of pressure from the start pad until contact with the target pad). The primary dependent variable in this investigation was RT which was used to determine if the processing speed of directional arm movements can be facilitated by teaching children lateral exergame maneuvers. As indicated, MT was recorded but the scores were only used to 
provide participants a source of motivation to encourage them to perform each trial as fast as possible.

\section{Testing Procedures}

Before any data were collected each participant engaged in a brief orientation session to familiarise the participant with the data collection procedures. Instructions given during this session were standardised for each participant. During this brief session participants practiced each of the directional movements three times with each extremity. During these six practice movements, feedback was provided by the experimenter to ensure each participant felt comfortable with the procedures.

To begin, each participant was required to sit during testing to establish a midline in the seated position. Participants sat at a table with their xiphoid process aligned with the start pad and the midline target position. Table height was individually adjusted to accommodate the height of each child. Data were collected by having the participant perform goal-directed aiming movements with each arm in separate trial blocks, with the order of start arm counterbalanced across participants. Reaching movements from the start pad to target pad were made away from the body primarily involving extension of the elbow. Within a trial block, the participant completed nine trials to each of the three target positions (i.e., contralateral, midline, and ipsilateral) and three catch trials, based on stratified random presentation. The three catch trials were embedded within a block of empirical trials to invoke an uncertainty of occurrence. Once the randomly selected target stimulus was illuminated the participant was instructed to move his/her arm to the corresponding target pad as quickly and accurately as possible. When the catch trial stimulus illuminated the participant was instructed to not move from the start pad.

Before a trial was initiated, the participant was asked to attend to a fixation point at the center of the apparatus. Once attention was acknowledged, the warning buzzer was 
triggered and then a randomly selected foreperiod, of either 1.5, 3.0, or 4.5 seconds, preceded the onset of the target stimulus LED to prevent anticipation of the stimulus. The importance of the centrally located fixation point was stressed and monitored throughout the experiment. When the participant failed to maintain fixation during the foreperiod these trials were noted, discarded, and replaced at the end of each block of trials. These occurrences were rare and did not differ significantly between the groups of children. During the pre-test each participant performed a total of 54 empirical trials of goal-directed aiming movements with the left and right arms. A post-test, involving the same response time data collection procedures, was repeated after the 30-minute treatment accumulating 108 upper extremity response time trials for each participant.

\section{Treatment Procedures}

In between pre and post response time assessments each participant performed 30 minutes of treatment in one of three groups (WTC, WBI, control). This short treatment session was utilised to replicate the treatment time used in the previous experiment (Pedersen 2014). All treatment sessions were led by the first author in an isolated room that was free of furniture except for a one metre tall television cart that contained the Nintendo Wii gaming console on a shelf, and a 32" LCD television placed on top of the cart (this cart was removed for the control sessions). Before treatment each participant was provided a five-minute instruction period to become familiar with the exergame/control activities where correct body movements were demonstrated to ensure the participant would experience a level of success.

Children in the WTC group were exposed to deliberate practice involving two-handed (bimanual) and two-legged tennis groundstroke movements using the Wii controller in place of a real tennis racquet. During the 30 minutes of treatment time these participants were required to perform contralateral arm and leg movements in order to successfully 'hit' twohanded, forehand and backhand tennis shots that only required midline crossing manoeuvres. 
The serving action was omitted during this protocol. Participants were first instructed to await the oncoming tennis shots delivered by the computer player by positioning their body in the ready position: standing with their feet shoulder width apart, knees slightly bent, eyes and shoulders facing the computer screen, and holding the Wii controller with both hands (right hand superior to left hand) directly in front of their umbilicus. During play, when the computer game indicated that the participant needed to hit a forehand shot to return the ball across the net, the participant was instructed to initiate the shot by stepping the left foot anterolaterally across the midline into the contralateral space. Simultaneously, they were instructed to move both hands (while holding the Wii controller just as s/he would hold the grip of a tennis racquet) to the right side of the body while rotating the hips to the right to prepare the forehand stroke. In an effort to make contact with the imaginary ball the participant then was told to swing both hands anteriorly on the right side of the body with a follow through motion that crossed back across the rotated midline of the body resulting in both hands (and Wii controller) on the left side of the body. This sequence was a mirror image on the left side of the body when a backhand shot was required. All shots (forehand and backhand) required three midline crossing movements: 1) stepping across the midline anterolaterally with opposite foot to prepare a 'hit' on the side of the oncoming ball, 2) preparing the hands (one across the midline) to initiate a shot on the side of the oncoming ball; and 3) and swinging the hands (and Wii controller) across the midline anteriorly during the 'hitting' action. Once each swing was completed the participant was instructed to return to the ready position and await the next shot made by the computer. Groundstroke frequency was recorded for each participant in this group. An equal amount of forehand strokes and backhand strokes were made during the treatment session across all participants in this group.

Children in the WBI group were exposed to deliberate practice involving two-handed (unimanual) and two-legged ipsilateral bowling movements using the Wii controller in place 
of a real bowling ball. During the 30 minutes of treatment time these participants were required to perform ipsilateral arm and leg movements in order to successfully simulate 'bowling' an imaginary bowling ball that only required non-midline crossing movements. That is, all steps and under-arm swinging motions were made in the ipsilateral space through the frontal plane only. The bowling game required the participants to bowl 10 frames as in a typical bowling game. For the purposes of the study, participants were told to bowl the odd frames with their right hand, and to bowl the even frames with their left hand. This ensured that ipsilateral movements were evenly dispersed among both hands during the treatment session, as was accomplished in the WTC group training. Before the gameplay began the participants were instructed how to perform a three-step bowling approach, release, and follow through while maintaining ipsilateral movements that did not involve any midline crossing movements.

The children in the control group did not perform any arm movement exercise; instead they played a hand-held video game on the Nintendo DS while standing. These participants were instructed to not move around, so only bilateral thumb movements that did not cross the midline were elicited during this 30 minute control treatment.

To address the physical fidelity of the desired transfer effect, the goal of the experimental treatments was to improve the lateral arm movement skills of the participant through deliberate practice. More specifically, the WTC group was deliberately taught to become more efficient at contralateral arm movements, whereas the WBI group was trained to improve purposeful ipsilateral arm movements in order to experience success at the respective exergames. To accomplish these goals each training session was structured to only allow for these movements during the gameplay. If the participant was seen making incorrect movements the game (and clock) was stopped and the participant was re-directed to perform the correct movements with verbal instructions only. These instances were infrequent, and 
never required demonstration or physical guidance. The third author who was not involved in leading the treatment sessions was able to record academic learning time - physical education (ALT-PE) by taking brief notes regarding the on and off participation times on a notepad while utilising a clock on the wall in the testing room. Participants averaged $26+/-2.38$ minutes of ALT-PE throughout the study. An entire data collection session (including pretest, treatment, and post-test) lasted approximately 90 minutes. There was no evidence of fatigue by any of the children in this study.

\section{Data Analysis}

In retrospect of the previous investigation (Pedersen 2014), we decided to omit the side of body (right, left) independent variable from this investigation since it previously produced no significant effects. Instead, the reviewers from our previous paper suggested that it may be interesting to investigate an age range effect to note any developmental differences. Thus, in the current investigation we decided to manipulate our sample to test younger (7-9 year olds) and older (10-12 year olds) cohorts to determine if developmental differences contributed to our findings.

Prior to any analyses, RT and MT scores were analysed using Pearson Product Moment Correlation coefficients $(r)$ to determine any relationships that may exist. The relationships between RT and MT for the three treatment groups across pre- and post-tests, and all three directions were low. The correlation coefficients ranged from $r=-0.05$ to $r=0.18$. Since these variables were statistically independent we decided to inferentially test RT as a measure of processing extremity movements using an analysis of variance (ANOVA) procedure.

The dependent variable was subject to a frequency distribution analysis for each participant within each block of trials to determine normality. Values that landed outside of the $+/-3$ standard deviation range were declared outliers and were replaced with the mean 
score of the nine trials of that particular trial block. These outlying scores represent anticipations or lapses in attention (Clarke and Zaidel 1989), and confound attempts to measure information processing in children. Less than $2 \%$ of all trials were found to represent outliers. The sphericity assumption was fulfilled within these data. Reliability estimates using intraclass correlation coefficients were calculated for the dependent variable across each level of all independent variables. All statistical analyses were based on trial level data.

Independent variables manipulated in this study included treatment group (WTC, WBI, control), age group (7-9 year olds, 10-12 year olds), test (pre-test, post-test), and movement direction (contralateral, midline, ipsilateral). Test and movement direction were the only repeated measures. Alpha levels were set a priori to 0.05 . When appropriate significant interactions were further examined using simple main effects analyses, and post hoc comparisons were performed using Tukey's honestly significant difference (HSD) test.

\section{Results}

\section{Reliability}

The reliability coefficients for RT across the three treatment groups ranged between 0.87 and 0.92 . This finding is in accordance with past investigations of reliability using this apparatus to assess choice RT in children (Woodard and Surburg 1999; Pedersen et al. 2004;

Pedersen 2014).

\section{Inferential findings}

A significant age group by treatment group by direction triple interaction, $F(4,48)=2.96, p<0.05$, was found. However, the only significant simple main effects were evident between the two age groups, indicating that the older group of children were significantly faster than the younger children across all three treatment groups and all three directions, $F(1,54)$ values ranged between $39.85-175.92, p<0.05$. There were no significant 
simple main effect findings between treatment groups for all directions within age groups, or between directions for all treatment groups within age groups. Age group means and standard deviations can be viewed in Table 1. [Table 1 near here]

This age group finding was further supported by a significant interaction between age group and direction, $F(2,48)=5.23, p<0.05$. Simple main effects for this analysis also indicated that the younger group had significantly slower RT scores compared to the older group for all three directional scores, $F(1,54)$ values ranged between $203.03-327.70$, $p<0.05$; whereas there were no statistically significant directional differences noted within each age group, $F(2,52)$ values ranged between $0.08-0.36, p>0.05$. Once again this was supported by a main effect for age group, $F(1,24)=17.50, p<0.05$, with the older group ( $M=353, S D=145 \mathrm{~ms}$ ) having significantly faster arm RT compared to the young group $(M=479, S D=208 \mathrm{~ms})$; regardless of treatment, test, or direction. These findings support a wealth of literature that as children develop through childhood their reaction times improve (e.g., Eckert and Eichorn 1977). This was further supported by the smaller standard deviations exhibited by the older group across all three treatment groups and all three directions when compared to the younger group.

Notably, there were no significant findings regarding the treatment effects of the preand post-test RT scores even when age group data were collapsed, $F(4,48)=1.11, p=0.36$. To illustrate the comparative nature of our report in Table 2 we have compared mean difference data from our current study using the exergame treatments to our previous study (Pedersen 2014) that utilised traditional physical activity training. The only procedural differences between these two studies were the type of contralateral and ipsilateral training groups used in the treatment. Exergames were used in the current study, whereas ball bouncing games were used in the previous work. [Table 2 near here]

\section{Discussion}


The most important finding in our study was a non-significant one, a rejection of our hypothesis. That is, a short-bout of deliberate laterality practice using exergames did not improve the motor processing speed of lateral arm movements in the same manner of our previous experiment (Pedersen 2014) using ball bouncing and catching games as the treatment. This suggests that using a commercially available virtual game environment (e.g., Nintendo Wii) might not be as effective for improving psychomotor development in children compared to more traditional physical activity instruction. Physical educators who gravitate towards this type of instruction may be drawn to the motivational factors children associate with video game play, but if there are low amounts of physical and cognitive fidelity in this training method then positive transfer to the desired skill acquisition is unlikely. Moreover, playing exergames as a means of skill development during physical education instruction might result in the development of poor motor habits. Once learned, skill habits are difficult to correct and hence it becomes increasing difficult to improve. Thus, with good intentions of decreasing sedentariness in young people, the inclusion of exergames at the expense of high physical and cognitive fidelity training instruction might unwittingly contribute to their sedentariness.

Unfortunately there is a lack of literature in this regard to compare our findings to. We propose a few possible explanations as to why exergames may be less than ideal for psychomotor development in children. Exergames can benefit children in terms of increasing energy expenditure (e.g., Miyachi et al. 2010), but similar improvements in cognitive processing associated with movement have not been supported. For example, O’Leary et al. (2011) found that short bouts of exergame play were unable to generate the increased allocation of attentional resources during a flanker task in the same manner of short bouts of aerobic exercise. While these results were interesting, no speculative reasoning as to why this occurred was offered in their paper. The importance of cognitive functioning as a 
contributory factor to the processing speed of movement execution is well understood. The time in which a movement is initiated can contribute to the success or failure of a given movement trial (McLeod 1987). Thus, comparing exergames to traditional physical activity to facilitate psychomotor development might be constrained by the cognitive functioning required for these two types of game play. For example as suggested by O'Leary and colleagues the attentional focus required for games that involve actual movements might require greater neurological resources compared to the pseudo-movements necessary for success during video gameplay. Exergames allow for a greater margin for error to produce successful outcomes in a virtual environment. This greater range might permit for slower processing demands on the player to still elicit a successful outcome on the screen. Whereas, the same slow processing speeds will result in a failed catch when playing a ball bouncing game that requires the player to organise several muscles to produce both gross and fine motor movements with precision to be successful during a catch. This attribution of attentional focus towards successful gameplay might be one reason for the disparate findings of our two laboratory studies.

Augmented feedback obtained during game play, both virtual and real, can facilitate skill development. Exergames are designed to make this virtual feedback seem as real as possible through visual graphics, auditory prompts, and haptic feedback (e.g., vibrations made by the hand-held game controller). Nonetheless, moving the exergame controller through space does not replicate the physical fidelity associated with manipulating the actual object. Performing movements with actual sporting equipment allows for greater variability in proprioceptive feedback from the extremities (van Beers, Wolpert, and Haggard 2002). For example, at present there is little resemblance between a gaming and a training situation for the physical fidelity of hitting a well-timed tennis shot versus a poorly timed tennis shot. However, the exergame controller that attempts to approximate this feeling during a tennis 
exergame is limited in the haptic cues it can provide the user's hand for central nervous system processing. This additional neural processing experienced during the ball bouncing games might have strengthened the neural pathways responsible for processing the lateral movements during the RT testing. Whereas the lack of this proprioceptive input during exergame play might have inhibited the effect of the treatment on improving movement initiation speed.

Another important factor in skill development is the ability to tailor activities during training to the unique needs of the learner (Reilly et al. 2009). The ability to individualise the training sessions was more evident during the ball bouncing games compared to the exergames. The ball bouncing games used in Pedersen (2014) allowed for immediate increases in task difficulty through the 30 minute training session as soon as proficiency was attained by each participant. In contrast, exergame training constrained by programming code only elicits repetitive movement skills and only allows for advancement to more difficult stages once the present stage is completed. This code is hard wired, and the time for each stage cannot be manipulated by the research team or the user. This difference prohibits the physical educator from providing differentiated training experiences for individuals of differing abilities. Thus the exergame environment is still a 'one size fits all' experience that cannot yet cater for modifying tasks to move an individual from one level to the next. The individualised challenges and corrective skill feedback provided to participants during the ball bouncing games appear to have facilitated skill acquisition. This teacher-centered deliberate movement practice resulted in significant improvements in lateral arm and leg movement processing (Pedersen 2014), whereas the absence of a teacher figure during the exergame training might have resulted in our insignificant findings. Although exergames may be fun and engaging, they have reduced individualisation abilities when compared to traditional physical activity. This lack of individualisation might have resulted in skill levels 
being more or less maintained throughout the 30 minute exergame training session once they were attained.

Similar to our previous work, there were limitations in our current study. It was our goal to replicate the previous work as closely as possible, thus limitations regarding sample size, motor skill learning measurement, and treatment length were not addressed. We recommend that future research consider each of these while utilising a more complex, perhaps cross-over, research design to decipher the differences between traditional physical activity and exergame play in regards to the skill transfer of psychomotor skill development in a real world context. This type of study will be able to address the ecological relevance of our reaction time findings to actual skill development. In the meantime, we propose caution should be used in not only interpreting our findings from the two investigations, but also caution should be adhered to by health and physical education teachers before they choose to use exergame strategies in lieu of traditional physical activity instruction.

\section{Summary for Practitioners}

In conclusion, using exergames to deliver deliberate laterality practice we were unable to replicate the significantly positive transfer effect on the sensory-motor processing of lateral movements produced by traditional physical movements. Therefore we recommend that schools should not substitute exergames for traditional instruction from qualified health and physical education teachers. This is primarily because any health benefits of using exergaming are negated by students not making comparable psychomotor improvements due to a lack of engagement with the brain. Physical education is not just about energy expenditure, there is also skill development and cognitive benefits that occur during traditional game play that is not achieved through simulated training with commercially available exergames. We support the use of exergames to supplement physical education programs, or to substitute for sedentary activities whilst at home. While we acknowledge 
previous research stating the potential benefits of exergames, we feel that these benefits are best achieved, as Staiano and Calvert (2011) state, as engaging, and health-promoting homework assignments. 


\section{References}

Australian Curriculum and Reporting Authority (ACARA). (2015). Australian Health and Physical Education Curriculum. Retrieved from: http://www.australiancurriculum.edu.au/health-and-physical-education/

Bailey, B. W., \& McInnis, K. (2011). Energy cost of exergaming: A comparison of the energy cost of 6 forms of exergaming. Archives of Pediatrics and Adolescent Medicine, 165(7), 597-602.

Bisson, E., Contant, B., Sveistrup, H., \& Lajoie, Y. (2007). Functional balance and dual-task reaction times in older adults are improved by virtual reality and biofeedback training. Cyberpsychology and Behavior, 10(1), 16-23.

Brumels, K. A., Blasius, T., Cortright, T., Oumedian, D., \& Solberg, B. (2008). Comparison of efficacy between traditional and video game based balance programs. Clinical Kinesiology, 62(4), 26-31.

Bryden, M. (1977). Measuring handedness with questionnaires. Neuropsychologia, 15(4), 617-624.

Chandler, T., Anthony, M., \& Klinger, D. (2009). High cognitive fidelity vs. high fidelity in serious games. Interservice/Industry training, Simulation, and Education Conference. Orlando, Florida.

Clarke, J., \& Zaidel, E. (1989). Simple reaction times to lateralized light flashes varieties of interhemispheric communication routes. Brain, 112(4), 849-870.

Eckert, H. M., \& Eichorn, D. H. (1977). Developmental variability in reaction time. Child Development, 48, 452-458.

Ericsson, K. A., Krampe, R. T., \& Tesch-Römer, C. (1993). The role of deliberate practice in the acquisition of expert performance. Psychological Review, 100(3), 363-406. 
Graf, D. L., Pratt, L. V., Hester, C. N., \& Short, K. R. (2009). Playing active video games increases energy expenditure in children. Pediatrics, 124(2), 534-540.

Hale, S., Fry, A. F., \& Jessie, K. A. (1993). Effects of practice on speed of information processing in children and adults: Age sensitivity and age invariance. Developmental Psychology, 29(5), 880-892.

Hansen, L., \& Sanders, S. (2008). Interactive gaming: Changing the face of fitness. Florida Alliance for Health, Physical Education, Recreation, Dance \& Sport Journal, 46(1), $38-41$.

Hayes, E., \& Silberman, L. (2007). Incorporating video games into physical education, Journal of Physical Education, Recreation, and Dance, 78(3), 18-24.

Hubert, B. (2001). Bal-A-Vis-X: Rhythmic balance/auditory/vision exercises for brain and brain-body integration. Wichita, KS: Bal-A-Vis-X.

Kail, R. (1991). Developmental change in speed of processing during childhood and adolescence. Psychological Bulletin, 109(3), 490-501.

Kliem, A., \& Wiemeyer, J. (2010). Comparison of a traditional and a video game based balance training program. International Journal of Computer Science in Sport, 9(3), $80-91$.

Lager, A., \& Bremberg, S. (2005). Health effects of video and computer game playing: A systematic review. Stockholm: Swedish National Institute of Public Health (Translation of Swedish NIPH Report 2005:18, ISSN: 1651-8624).

Marshall, S. J., Biddle, S. J., Gorely, T., Cameron, N., \& Murdey, I. (2004). Relationships between media use, body fatness and physical activity in children and youth: A metaanalysis. International Journal of Obesity and Related Metabolic Disorders, 28(10), $8-46$. 
McLeod, P. (1987). Visual reaction time and high-speed ball games. Perception, 16(1), 4959.

Miyachi, M., Yamamoto, K., Ohkawara, K., \& Tanaka, S. (2010). METs in adults while playing active video games: A metabolic chamber study. Medicine and Science in Sports and Exercise, 42(6), 1149-1153.

O’Leary, K. C., Pontifex, M. B., Scudder, M. R., Brown, M. L., \& Hillman, C. H. (2011). The effects of single bouts of aerobic exercise, exergaming, and videogame play on cognitive control. Clinical Neurophysiology, 122(8), 1518-1525.

Pedersen, S. J. (2014). Deliberate laterality practice facilitates sensory-motor processing in developing children. Physical Education and Sport Pedagogy, 19(2), 136-148.

Pedersen, S. J., Surbung, P. R., \& Brechue, W. (2005). Ageing and midline crossing inhibition. Laterality, 10(3), 279-294.

Pedersen, S. J., Surburg, P. R., Heath, M., \& Koceja, D. M. (2004). Fractionated lower extremity response time performance in boys with and without ADHD. Adapted Physical Activity Quarterly, 21(4), 315-329.

Reilly, T., Morris, T., \& Whyte, G. (2009). The specificity of training prescription and physiological assessment: A review. Journal of Sport Sciences, 27, 575-589.

Sheehan, D. P., \& Katz, L. (2013). The effects of a daily, 6-week exergaming curriculum on balance in fourth grade children. Journal of Sport and Health Science, 2(3), 131-137.

Staiano, A. E., \& Calvert, S. L. (2011). Exergames for physical education courses: Physical, social, and cognitive benefits. Child development perspectives, 5(2), 93-98.

Sun, H. (2012). Exergaming impact on physical activity and interest in elementary school children. Research Quarterly for Exercise and Sport, 83(2), 212-220.

van Beers, R. J., Wolpert, D. M., \& Haggard, P. (2002). When feeling is more important than seeing in sensorimotor adaptation. Current Biology, 12, 834-837. 
Vernadakis, N., Gioftsidou, A., Antoniou, P., Ioannis, D., \& Giannousi, M. (2012). Impact of Nintendo Wii to physical education students' balance compared o the traditional approaches. Computers and Education, 59(2), 196-205.

Widman, L. M., McDonald, C. M., \& Abresch, R. T. (2006). Effectiveness of an upper extremity exercise device integrated with computer gaming for aerobic training in adolescents with spinal cord dysfunction. The Journal of Spinal Cord Medicine, 29(4), 363-370.

Woodard, R. J., \& Surburg, P. R. (1999). Midline crossing behavior in children with learning disabilities. Adapted Physical Activity Quarterly, 16(2), 155-166.

Woollacott, M., \& Shumway-Cook, A. (2002). Attention and the control of posture and gait: a review of an emerging area of research. Gait and Posture, 16(1), 1-14.

Yang, S., Smith, B., \& Graham, G. (2008). Healthy video gaming: Oxymoron or possibility. Journal of Online Education, 4(4). Retrieved from: http://innovateonline.info/pdf/vol4_issue4/Healthy_Video_Gaming_Oxymoron_or_P ossibility_.pdf 
Figure 1 Schematic of Choice Response Time Apparatus

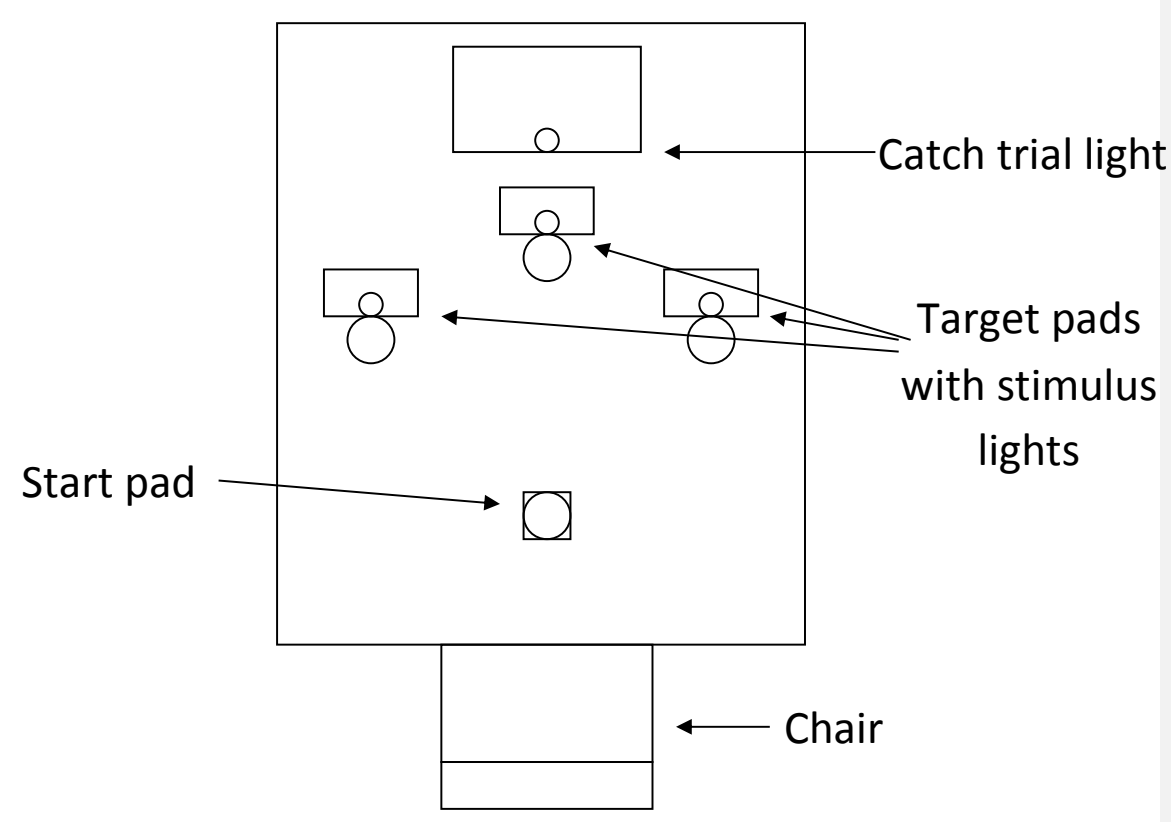


Table 1. Upper Extremity Reaction Time Means (Standard Deviations) for Age Group as a Function of Treatment Group and Direction.

Values are denoted in milliseconds. Directions are contralateral (Contra), midline (Mid), and ipsilateral (Ipsi). Treatments are the Wii Tennis

Contralateral (WTC) group and the Wii Bowling Ipsilateral (WBI) group. Each reaction time value for the 10-12 year olds was significantly faster than the corresponding value for the 7-9 year olds.

\begin{tabular}{|c|c|c|c|c|c|c|}
\hline & \multicolumn{3}{|c|}{$7-9$ year olds } & \multicolumn{3}{|c|}{$10-12$ year olds } \\
\hline Treatment / Direction & Contra & Mid & Ipsi & Contra & Mid & Ipsi \\
\hline WTC Group & 557 (159) & $518(143)$ & $507(131)$ & $391(88)$ & $388(85)$ & $410(104)$ \\
\hline WBI Group & $512(126)$ & $507(115)$ & $498(121)$ & $404(85)$ & $408(90)$ & $403(77)$ \\
\hline Control Group & $590(163)$ & $546(143)$ & 550 (139) & $389(86)$ & $373(84)$ & $368(87)$ \\
\hline
\end{tabular}


Table 2. Upper Extremity Reaction Time Mean Difference Scores Between Pre-test and Post-test as a Function of Treatment Group and

Direction.

Values are denoted in milliseconds. Directions are contralateral (Contra), midline (Mid), and ipsilateral (Ipsi). Treatments are the Wii Tennis

Contralateral (WTC) group and the Wii Bowling Ipsilateral (WBI) group. A positive value indicates an improvement in reaction time as a function of the treatment.

${ }^{\mathrm{a}}$ indicates a significant improvement in reaction time. ${ }^{\mathrm{b}}$ indicates a significant decline in reaction time.

\begin{tabular}{|c|c|c|c|c|c|c|}
\hline \multirow[b]{2}{*}{ Treatment / Direction } & \multicolumn{3}{|c|}{$\begin{array}{l}\text { Mean Difference using Exergames } \\
\text { (Current Study) }\end{array}$} & \multicolumn{3}{|c|}{$\begin{array}{l}\text { Mean Difference using Ball Bouncing } \\
\text { (Pedersen, 2014) }\end{array}$} \\
\hline & Contra & Mid & Ipsi & Contra & Mid & Ipsi \\
\hline WTC group & -9 & -4 & 15 & $37^{\mathrm{a}}$ & 12 & $-17^{\mathrm{b}}$ \\
\hline WBI group & -9 & 15 & 9 & 2 & 11 & $28^{\mathrm{a}}$ \\
\hline Control Group & -10 & -6 & -9 & $-31^{b}$ & -4 & 2 \\
\hline
\end{tabular}

